\title{
35. COMMISSION DE LA CONSTITUTION DES ETOILES
}

Président: Sir A. S. Eddington, Director of the University Observatory, Cambridge, England.

Mzmbres: MM. Emden, R. H. Fowler, Jeans, Milne, Pannekoek, Rosseland, Russell, B. Strömgren, Vogt, Von Zeipel, Woltjer.

The Commission has found no opportunity for acting collectively during the last three years. The study of the constitution of the stars has been advanced by individual contributions, which it would be impracticable and undesirable to guide officially.

Whilst much useful work has been done, it would be difficult to point to any outstanding development in the study of the interior of the stars during this period. But there is a prospect of great advances in the near future. From I932 onwards experimental physicists in all countries have been largely occupied with the phenomena of transmutation of the elements, and it is clearly possible to determine in the laboratory most of the quantitative data as to the rate of the subatomic processes (especially those due to the encounters of protons and electrons with nuclei) and the consequent liberation of energy, for which astronomers have long been waiting.

The writer has recently contended that the "relativistic" degeneracy formula extensively used in stellar investigations is unsound and that the "ordinary" formula is the correct deduction from relativity and quantum theory. A decision on this point profoundly affects the theory of super-dense stars.

\author{
A. S. EDDington \\ President of the Commission
}

